\title{
199 THE INFLUENCE OF NICOTINAMIDE ON THE DEVELOPMENT OF NEURONS
}

Síle Griffin, ${ }^{1}$ Mark Pickard, ${ }^{1}$ Rowan Orme, ${ }_{1}^{1}$ Clive Hawkins, ${ }^{2}$ Adrian Williams, ${ }^{3}$ Divya Chari, ${ }^{1}$ Rosemary Fricker ${ }^{1} .{ }^{1}$ Institute for Science and Technology in Medicine, Keele University; ${ }^{2}$ Regional Neuroscience Centre, University Hospital of North Staffordshire; ${ }^{3}$ Divisions of Neurosciences and Medical Sciences, University of Birmingham

10.1136/jnnp-2014-309236.199

A major challenge in translating the promise of stem cell therapies to treat a myriad of neurodegenerative disorders is to rapidly and efficiently direct pluripotent stem cells to generate differentiated neurons. The application of active vitamin metabolites known to function in embryonic development and maintenance in the adult brain such as retinoic acid (vitamin A), ascorbic acid (vitamin C) and calcitriol (vitamin D3) have proven effective in current in-vitro differentiation protocols.

Therefore, in this study we investigated whether the biologically active vitamin B3 metabolite, nicotinamide could enhance the differentiation of mouse embryonic stem cells, cultured as monolayers, into mature neurons at either early or late stages of development.

Interestingly, nicotinamide elicited a dose-responsive increase in the percentage of neurons when added at an early developmental stage to the cells undergoing differentiation (days 0-7). Nicotinamide $(10 \mathrm{mM})$ increased the proportion of $\beta$-III tubulin positive neurons by two fold and concomitantly decreased the total number of cells in culture, measured by quantification of 4', 6-diamidino-2-phenylindole positive cells. This effect could result from induction of cell-cycle exit and/or selective cell death in non-neural populations. Higher levels of nicotinamide (20 $\mathrm{mM})$ induced cytoxicity and cell death.

This study supports previous evidence that vitamins and their metabolites can efficiently direct stem cells into neurons. Current work is focusing on the effect of nicotinamide on the process of neural induction and whether nicotinamide influences the generation of particular neuronal subtypes implicated in neurodegenerative diseases, specifically focusing on midbrain dopamine neurons; towards a therapy for Parkinson's disease. 